https://helda.helsinki.fi

\title{
When are Antiaromatic Molecules Paramagnetic?
}

\section{Valiev, Rashid R.}

2020-09-24

Valiev , R R , Baryshnikov , G V , Nasibullin , R T , Sundholm , D \& Ågren , H 2020 , ' When are Antiaromatic Molecules Paramagnetic? ' , Journal of Physical Chemistry C , vol. 124 , no. 38 , pp. 21027-21035 . https://doi.org/10.1021/acs.jpcc.0c01559

http://hdl.handle.net/10138/333373

https://doi.org/10.1021/acs.jpcc.0c01559

acceptedVersion

Downloaded from Helda, University of Helsinki institutional repository.

This is an electronic reprint of the original article.

This reprint may differ from the original in pagination and typographic detail.

Please cite the original version. 


\section{When are antiaromatic molecules paramagnetic?}

3 Rashid R. Valiev, ${ }^{a, b, *}$ Glib V. Baryshnikov,${ }^{c, d,}{ }^{*}$ Rinat T. Nasibullin, ${ }^{e}$ Dage Sundholm, ${ }^{b}$ and

4 Hans Ågren ${ }^{f, c}$

$6 \quad{ }^{a}$ Research School of Chemistry \& Applied Biomedical Sciences, National Research Tomsk

7 Polytechnic University, Lenin Avenue 30, Tomsk 634050, Russia

$8{ }^{b}$ Department of Chemistry, Faculty of Science, University of Helsinki, FIN-00014, Hel-

9 sinki, Finland

$10{ }^{c}$ Division of Theoretical Chemistry and Biology, School of Engineering Sciences in Chem-

11 istry, Biotechnology and Health, KTH Royal Institute of Technology, 10691, Stockholm,

12 Sweden

$13{ }^{d}$ Department of Chemistry and Nanomaterials Science, Bohdan Khmelnytsky National

14 University, 18031, Cherkasy, Ukraine

$15{ }^{e}$ Tomsk State University, Lenin Avenue 36, Tomsk 634050, Russia

$16{ }^{f}$ College of Chemistry and Chemical Engineering, Henan University, Kaifeng, Henan 17 475004, P.R. China

19 ABSTRACT: Magnetizabilities and magnetically induced current densities have been cal20 culated and analyzed for a series of antiaromatic cyclo[4k]carbons $(\mathrm{k}=2-11)$, iso[n]phlorins 
$1 \quad(\mathrm{n}=4-8)$, expanded porphyrinoids and meso-meso, $\beta-\beta, \beta-\beta$ triple linked porphyrin and iso-

2 phlorin arrays. The cyclo[4k]carbons with $\mathrm{k}=2-6$ are predicted to be closed-shell paramag-

3 netic molecules due to the very strong paratropic ring current combined with its large radius.

4 Larger cyclo[4k]carbons with $\mathrm{k}=6-11$ are diamagnetic, because they sustain a paratropic

5 ring current whose strength is weaker than $-20 \mathrm{nA} \mathrm{T}^{-1}$, which seems to be the lower threshold

6 value for closed-shell paramagnetism. This holds not only for cyclo[4k]carbons, but also

7 for other organic molecules like expanded porphyrinoids and oligomers of porphyrinoids.

8 The present study shows that meso-meso, $\beta-\beta, \beta-\beta$ triple linked linear porphyrin and iso-

9 phlorin arrays have a domain-like distribution of alternating diatropic and paratropic ring

10 currents. The strength of their local paratropic ring currents is weaker than $-20 \mathrm{nA} \mathrm{T}^{-1}$ in

11 each domain. Therefore, linear porphyrin and isophlorin arrays become more diamagnetic

12 with increasing length of the ribbon. For the same reason, square-shaped meso-meso, $\beta-\beta$,

$13 \quad \beta-\beta$ triple linked free-base porphyrin and isophlorin tetramers as well as $\mathrm{Zn}$ (II) complex of

14 the porphyrin tetramer are diamagnetic. We show that closed-shell molecules with large

15 positive magnetizabilities can be designed by following the principle that a strong para-

16 tropic current ring combined with a large ring-current radius leads to closed-shell paramag-

17 netism.

\section{1. INTRODUCTION}

20 The aromaticity concept is manifested by molecular structure, ${ }^{1}$ chemical reactivity, ${ }^{2}$ spec21 troscopic properties, ${ }^{3}$ magnetic properties, ${ }^{4}$ and molecular conductivity. ${ }^{5}$ A large number of 
1 studies have shown that the degree of aromatic-city can be determined from magnetic

2 shieldings,${ }^{6}$ ring-current strengths ${ }^{7}$ and other molecular magnetic properties. ${ }^{8-11}$ Electron de-

3 localization and pathways of magnetically induced current densities provide information

4 about molecular behaviour in the presence of an external magnetic field (B), which is im-

5 portant when designing novel magnetic materials. ${ }^{12}$ The magnetic susceptibility $(\chi)$ also

6 called magnetizability is a measure of interactions between matter and an external magnetic

7 field (B). ${ }^{13}$ The magnetizability $(\chi)$ is a second-rank tensor that can be formally divided into

8 diamagnetic $\left(\chi^{d}\right)$ and paramagnetic $\left(\chi^{p}\right)$ contributions. ${ }^{13-17}$ The magnetically induced current

9 density (J), which is the first-order electronic response to an external magnetic field (B),

10 can analogously be decomposed into diamagnetic $\left(\mathbf{J}_{\text {dia }}\right)$ and paramagnetic $\left(\mathbf{J}_{\text {para }}\right)$ current-

11 density contributions. ${ }^{16}$

12 Since most closed-shell organic compounds are non-aromatic or aromatic, $\chi^{p}$ is in absolute

13 value often smaller than $\chi^{d}$. The magnetizability of organic molecules is then diamagnetic

14 corresponding to negative magnetizability values, by definition. The more negative mag-

15 netizability, the stronger diamagnetic is the molecule. When $\chi^{p}$ dominates, the molecule is

16 paramagnetic with a positive magnetizability. Paramagnetic molecules are in general open-

17 shell molecules, whereas closed-shell paramagnetism (CSP) is rare. ${ }^{13,18}$ Diatomic $\mathrm{BH}$ and

18 its isoelectronic analogues $\mathrm{CH}^{+}$and $\mathrm{BeH}^{-}$are known to be closed-shell paramagnetic mol-

19 ecules, ${ }^{19-21}$ whereas only a few examples of closed-shell paramagnetic polyatomic mole-

20 cules have been reported..$^{22,23}$ 
1 We have shown in a number of recent studies that antiaromatic molecules sustaining a

2 strong paratropic ring current can be closed-shell paramagnetic molecules, ${ }^{24-26}$ which are

3 spectroscopically characterized by intense low-lying magnetic-dipole-allowed electronic

4 transitions. Strong paratropic ring currents also affect the magnetizability, because the an-

5 gular momentum of the paratropic ring current increases the $\chi^{p}$ contribution. Anderson et.

$6 a l^{23}$ recently detected CSP for closed-shell multicharged antiaromatic porphyrin nanorings,

7 which experimentally confirmed that CSP can occur in strongly antiaromatic molecular

8 rings.

9 The article is outlined as follows. In the next section, we briefly discuss the theory of

10 closed-shell paramagnetism. In section 3, the employed computational methods are pre-

11 sented. These methods are applied in section 4.1 to a closed-shell paramagnetic tetra-cat-

12 ionic hexaporphyrin nanowheel, whose magnetizability has been measured experimen-

13 tally. In section 4.2, we study the magnetizability and ring-current strengths of a series of

14 antiaromatic cyclo[4k]carbon rings. In section 4.3 we discuss magnetic properties of por-

15 phyrinoid arrays, while the data for expanded isophlorins and expanded porphyrinoids are

16 presented in sections 4.4 and 4.5 , respectively. The aromaticity of the square-shaped

17 [36]octaphyrin(1.1.1.1.1.1.1.1) and cyclo-octafuran(1.1.1.1.1.1.1.1) is discussed in section

18 4.6. The obtained results are summarized and concluded in section 5. 


\section{2. THEORY}

2 Molecular magnetizability is generally calculated as the second derivative of the electronic

3 energy with respect to the strength of the external magnetic field using the response formal-

4 ism. ${ }^{27-29}$ However, the mechanism of closed-shell paramagnetism can be easier understood

5 from the sum over states expression for the second derivative, where the magnetizability is

6 split into diamagnetic $\left(\chi^{d}\right)$ and paramagnetic $\left(\chi^{p}\right)$ contributions $^{30}$

$$
\begin{aligned}
\left.\chi_{\alpha \beta}=-\frac{1}{4} \sum_{i}\langle 0| r_{O i}^{2} \delta_{\alpha \beta}-r_{O i \alpha} r_{O i \beta}\right)|0\rangle \\
+\frac{1}{2} \sum_{\substack{i \\
K>0}} \frac{\left\langle 0\left|\left(\boldsymbol{r}_{O i} \times \boldsymbol{p}_{i}\right)_{\alpha}\right| K\right\rangle\left\langle K\left|\left(\boldsymbol{r}_{O i} \times \boldsymbol{p}_{i}\right)_{\beta}\right| 0\right\rangle}{E_{K}-E_{0}}
\end{aligned}
$$

9 The summation in Eq (1) runs over all electrons $i$ and excited states $K . \boldsymbol{r}_{O i}=\boldsymbol{r}_{i}-\boldsymbol{r}_{O}$ is

10 the distance from the common gauge origin, $\boldsymbol{p}_{i}$ is the momentum operator, and $E_{0}$ and $E_{K}$

11 are the energy of the ground and exited states, respectively. The diamagnetic term is always

12 negative and depends only on the ground state, whereas the paramagnetic part contains the

13 orbital angular momentum operator and is always positive. Low-lying excited states with

14 large magnetic transition moments from the ground state have large paramagnetic contri-

15 butions to the magnetizability. These excited states have also been found to be the reason

16 for the antiaromatic character of isophlorin and other antiaromatic porphyrinoids. ${ }^{26}$

17 Magnetizabilities can also be obtained by integrating the magnetically induced current den-

18 sity multiplied with the vector potential of the external magnetic field: ${ }^{16,31}$

$$
\chi_{\alpha \delta}=-\frac{1}{2 c} \varepsilon_{\alpha \beta \gamma} \int r_{\beta} J_{\gamma}^{B_{\delta}}(\boldsymbol{r}) \mathrm{d} \mathbf{r},
$$


1 where $\varepsilon_{\alpha \beta \gamma}$ is the Levi-Civita tensor, $r_{\beta}$ is the vector potential and $J_{\gamma}^{B_{\delta}}(\boldsymbol{r})$ is the magneti-

2 cally induced current density susceptibility that we call current density in the rest of this

3 article. When the external magnetic field is applied in $z$ direction, $\chi_{z z}$ is obtained as:

$4 \quad \chi_{z z}=-\frac{1}{2 c} \int\left(x J_{y}^{B_{z}}(\boldsymbol{r})-y J_{x}^{B_{z}}(\boldsymbol{r})\right) d \boldsymbol{r}$

5 When a planar aromatic molecule lies in the $x y$ plane, $J_{y}^{B_{z}}(\boldsymbol{r})$ flows in the positive $y$ direc-

6 tion for positive $x$ values and in the negative $y$ direction when $\mathrm{x}$ is negative, leading to a

7 negative contribution to $\chi_{z z}$. The second term in Eq. (3) is also negative for aromatic mol-

8 ecules. The paratropic ring current of antiaromatic molecules flows in the opposite direc-

9 tion and results in positive contributions to $\chi_{z z}$. The two contributions in Eq. (3) are large

10 for strong ring currents whose radius is large. For ring-shaped molecules the $\chi_{x x}$ and $\chi_{y y}$

11 elements of the magnetizability tensor are negative as for most molecules and they scale 12 nearly linearly with the size of the molecule as originally observed by Pascal. ${ }^{32}$ The average

13 molecular magnetizability is calculated as the trace of the magnetizability tensor:

$14 \quad \chi=\frac{1}{3}\left(\chi_{x x}+\chi_{y y}+\chi_{z z}\right)(4)$

15 implying that the paramagnetic $\chi_{z z}$ contribution of antiaromatic molecules in the $x y$ plane

16 is cancelled by the diamagnetic contributions from $\chi_{x x}+\chi_{y y}$. The magnetizability is pos-

17 itive when $\chi_{z z}$ is larger than the absolute value of $\chi_{y y}+\chi_{x x}$. We have previously shown

18 that porphyrinoids become closed-shell paramagnetic when the strength of the paratropic

19 ring current exceeds $-20 \mathrm{nA} \mathrm{T}{ }^{-1},{ }^{24}$ which holds for molecules with about the same radius of 20 the ring current as isophlorin. 


\section{3. COMPUTATIONAL METHODS}

2 The molecular structures were optimized at the density functional theory (DFT) level us-

3 ing the M06-2X exchange-correlation functional ${ }^{33}$ and the def2-TZVP basis set ${ }^{34}$. It is

4 known that this hybrid functional well describe magnetic properties and magnetizabili-

5 ties. ${ }^{35-36}$ The NMR shielding constants were calculated by using the Gauge Including

6 Atomic Orbital (GIAO) method ${ }^{37}$ at the M06-2X level of theory. These calculations were

7 performed for isolated molecules using the Gaussian 16 program package. ${ }^{38}$

8 The GIMIC program ${ }^{39,40}$ was used for calculation of the magnetically induced current

9 densities and the integrated current densities. The magnetically induced current densities

10 were calculated using density matrices obtained in the NMR shielding calculations and

11 basis-set data. The magnetic field was oriented perpendicular to the molecular plane. The

12 strengths of the current densities were obtained by numerical integration of the current

13 density passing a rectangular surface perpendicular to the molecule plane. The magnetiz-

14 ability was also calculated using GIMIC.

15 We performed state-specific complete active space self-consistent field (SS-CASSCF)

16 calculations for free-base porphyrin arrays $\left(\mathbf{H}_{2} \mathbf{P}\right)_{\mathrm{n}}$ and isophlorin arrays $(\mathbf{I P h})_{\mathrm{n}}$ with

$17 \mathrm{n}=1,2,3,4$. The SS-CASSCF calculations included 8 electrons in 8 molecular orbitals

18 (MO). The weight of closed shell determinant varies in the range of 0.9-0.93. For other

19 determinants, they are less than 0.15 implying that the studied molecules are single-refer-

20 ence systems. The SS-CASSCF calculations were carried out with the def2-TZVP basis

21 set using the Firefly software. ${ }^{41}$ 


\section{4. RESULTS AND DISCUSSION}

2 4.1 Hexaporphyrin nanowheel. The experimentally measured magnetizability of the re-

3 cently synthesized tetra-cationic hexaporphyrin nanowheel $\left(\mathbf{c}-\mathbf{P 6} \cdot \mathbf{T} \mathbf{6}^{\mathbf{4}}\right)$ in Figure 1 is 4418 a.u. ${ }^{23}$ Our calculations at the M06-2X/def2-TZVP level yield a magnetizability of 3188 a.u.

5 suggesting that DFT calculations using the M06-2X functional slightly underestimate the

6 magnetizability of the strongly antiaromatic molecules. Previous calculations on antiaro-

7 matic expanded porphyrins showed that density functionals with less than $50 \%$ Hartree-

8 Fock exchange tend to overestimate the strength of the paratropic ring current of strongly

9 antiaromatic molecules. ${ }^{42}$ A large positive magnetizability is obtained for $\mathbf{c - P 6} \cdot \mathbf{T 6}^{\mathbf{4 +}}$, be-

10 cause it sustains a strong paratropic ring current of $-65 \mathrm{nA} \mathrm{T}^{-1}$ whose radius is $13 \AA$. A larger

11 positive magnetizability can be obtained by either increasing the radius of the ring current

12 or by increasing the strength of the paratropic ring current. However, the diamagnetic con-

13 tribution to the magnetizability also increases with increasing size of the molecule accord-

14 ing to Pascal's rule. Closed-shell paramagnetism relies on the balance between the para15 magnetic contribution from $\chi_{z z}$ and the diamagnetic contributions from the $\chi_{x x}$ and $\chi_{y y}$ terms.

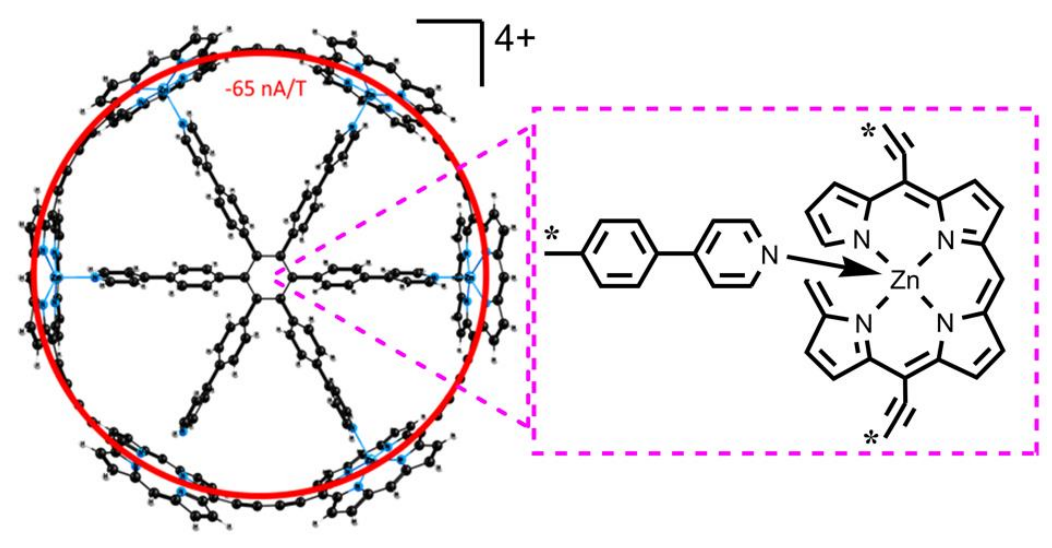


1 Figure 1. The molecular structure of the tetra-cationic hexaporphyrin nanowheel

$2\left(\mathbf{c}-\mathbf{P 6} \cdot \mathbf{T 6}^{\mathbf{4}}\right) \cdot{ }^{23}$ The paratropic ring current circulation pathway is schematically indicated

3 with the red ring with an average radius $13 \AA$.

$4 \quad 4.2$ Cyclo[n]carbons. The magnetizability and ring-current strengths have been also stud-

5 ied for a series of all-carbon molecules consisting of antiaromatic cyclo[n]carbons, i.e., cy-

6 clic $\mathrm{C}_{\mathrm{n}}$ polyynes ( $\mathrm{n}=4 k$, with $\left.k=2-11\right)$ that satisfy Hückel's rule for antiaromaticity. The

7 obtained results are summarized in Table 1. The calculations show that the strength of the

8 induced paratropic ring current decreases with increasing number of carbon atoms and be-

9 comes diatropic for the two largest all-carbon rings. Since the radius of the molecular ring

10 increases with the number of carbons, the product of the radius times the ring current

11 strength passes through a maximum for $\mathrm{n}=20(k=5)$, which also has the largest $\chi_{z z}$ value of

12105 a.u. The magnetizability is positive for $k=2-6$, whereas the larger polyynes are diamag-

13 netic, because $\chi_{x x}$ and $\chi_{y y}$ are negative and they increase in absolute value linearly with

14 the size of the molecule. The calculations show that cyclo[4k]carbons with $k=2-6$ are closed-

15 shell paramagnetic molecules. Cyclo[28]carbon is diamagnetic even though it has a radius

16 of $5.7 \AA$ and sustains a strong paratropic ring current of $-15 \mathrm{nA} \mathrm{T}^{-1}$, because the sum of the

17 diamagnetic contributions from $\chi_{x x}$ and $\chi_{y y}$ of -84 a.u. is larger than the paramagnetic $\chi_{z z}$

18 contribution of 67 a.u. Cyclo[44]carbon molecule, which is the largest all-carbon ring that

19 we studied, is slightly aromatic with a ring-current strength of $4 \mathrm{nA} \mathrm{T}^{-1}$ resulting in dia-

20 magnetism with a magnetizability of -59 a.u. 
1 Annulenes with $(4 \mathrm{k}+2) \pi$ electrons exhibit a more stable $\pi$ system with Hückel topology

2 and those with (4k) $\pi$ electrons prefer a Möbius twisted structure. ${ }^{43} \mathrm{Cyclo}[\mathrm{n}]$ carbons $(\mathrm{n}=4 \mathrm{k})$

3 become aromatic for large n values due to the change from Hückel to Möbius topology of

4 the conjugated $\pi$ electrons. Constructing Frost-Musulin mnemonic diagrams shows that cy-

5 clo[4n]carbons can be actually considered as double [n]annulenes, whose $\pi$ electrons are

6 oriented orthogonally to the molecular plane and in the plane, respectively. ${ }^{44-46}$ With in-

7 creasing ring size (n), the difference between the Frost-Musulin mnemonic of the Hückel

8 system (the vertex of the n-gon is its lowest point) and the one for the Möbius system (the

9 lowest edge is horizontal) becomes smaller leading to almost the same $\pi$-electron energy.

10 For $\mathrm{n}=36(\mathrm{k}=9)$ and $\mathrm{n}=40(\mathrm{k}=10)$, the cyclo[n]carbons are non-aromatic sustaining very

11 weak ring currents of -0.5 and $0.9 \mathrm{nA} \mathrm{T}^{-1}$, respectively, implying that the antiaromatic

12 Hückel (4k) and the aromatic Möbius (4k) structures are degenerate. For n=44 (k=11), Mö-

13 bius aromaticity clearly dominates over the Hückel antiaromaticity leading to a diatropic 14 ring current of $4.0 \mathrm{nA} \mathrm{T}^{-1}$.

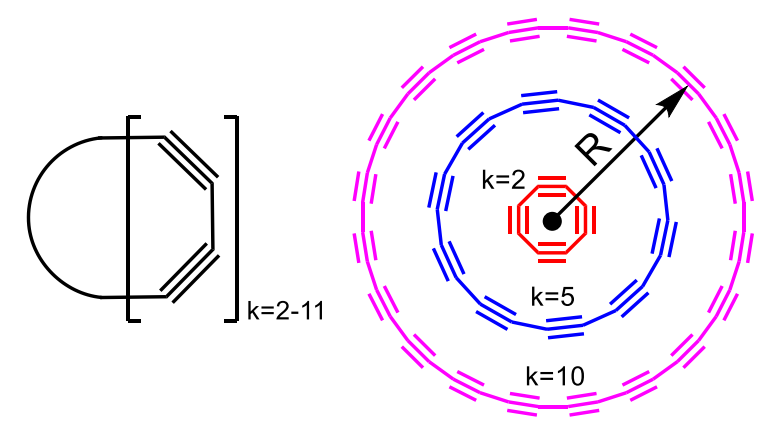

16 Figure 2. The formal molecular structures of the studied cyclo[n]carbons $(n=4 k)$. The 17 smallest studied molecule is cyclo[8]carbon $(k=2)$ and the largest one is cyclo[44]carbon $18(k=11)$. 
1 Table 1. The ring-current strength $\left(\mathrm{J}_{\text {in }} \mathrm{nA} \mathrm{T}^{-1}\right)$, the radius of the ring current ( $\mathrm{R}$ in $\AA$ ), and

2 the average magnetizability ( $\chi$ in a.u.) of the studied cyclo[n]carbons.

\begin{tabular}{llll}
\hline $\mathrm{n}$ & $\mathrm{J}$ & $\mathrm{R}$ & $\chi$ \\
\hline 8 & -42 & 1.8 & 5 \\
\hline 12 & -40 & 2.4 & 14 \\
\hline 16 & -36 & 3.5 & 18 \\
\hline 20 & -27 & 4.1 & 16 \\
\hline 24 & -18 & 4.9 & 7 \\
\hline 28 & -15 & 5.7 & -6 \\
\hline 32 & -4.4 & 6.5 & -20 \\
\hline 36 & -0.5 & 7.4 & -34 \\
\hline 40 & 0.9 & 8.4 & -47 \\
\hline 44 & 4.0 & 9.1 & -59 \\
\hline
\end{tabular}

4.3 Porphyrinoid arrays. Free-base porphyrin $\left(\mathrm{H}_{2} \mathbf{P}\right)_{0}$ is an aromatic and diamagnetic mol-

4 ecule with $\chi=-68$ a.u. ${ }^{24,25,47-49}$ It sustains a global diatropic magnetically induced ring cur-

5 rent of $27 \mathrm{nA} \mathrm{T}^{-1}$ by the 26 delocalized $\pi$ electrons. ${ }^{28}$ Isophlorin $(\mathbf{I P h})_{0}$ is chemically stable

6 and strongly antiaromatic sustaining a ring current of $-27 \mathrm{nA} \mathrm{T}^{-1}$ by its $28 \pi$ electrons. ${ }^{24}$

7 Isophlorin is weakly paramagnetic with a positive magnetizability of 12 a.u. at the M06-2X

8 level of theory and of 16 a.u. at the MP2 level. ${ }^{24}$ We have investigated how the magnetiz-

9 ability changes when extending porphyrin and isophlorin into the one-dimensional (1D)

10 meso-meso, $\beta-\beta, \beta-\beta$-linked arrays shown in Figure 3. In 2001, Osuka et al., synthesized

11 and fully characterized a meso-meso, $\beta-\beta, \beta-\beta$-linked porphyrin consisting of twelve $\mathrm{Zn}$ -

12 porphyrin $(\mathbf{Z n P})$ moieties $(\mathrm{n}=11)^{50}$. Eight years later same group synthesized fully conju-

13 gated porphyrin tapes consisting of $24 \mathrm{ZnP}$ moieties. $^{51}$

14 We have studied the current density of different moieties of the $1 \mathrm{D}(\mathbf{I P h})_{\mathrm{n}}$ arrays, because

15 total ring-current strengths are less meaningful. We found that the IPh arrays have a very 
1 unusual mixed aromatic-antiaromatic character. Analysis of the current-density pathways

2 in the $\mathbf{I P h}$ arrays indicates the presence of a strong almost independent paratropic ring cur-

3 rent of $-24 \mathrm{nA} \mathrm{T}^{-1}$ inside each IPh moiety. A weak diatropic ring current is sustained by the

4 naphthalene ring between the two IPh moieties. Longer $(\mathbf{I P h})_{\mathrm{n}}$ arrays $(\mathrm{n}=2-7)$ also sustain

5 localized paratropic ring currents in each IPh moiety. However, the paratropic ring currents

6 in the inner IPh fragments are somewhat weaker than the ones at the end of the IPh moie-

7 ties. The mixed aromatic-antiaromatic character suppresses the global electron delocaliza-

8 tion in the $(\mathbf{I P h})_{\mathrm{n}}$ arrays, which is supported by the almost size-independent HOMO-LUMO

9 gap (HLG) of the (IPh) $)_{\mathrm{n}}$ arrays (Table 2). Thus, there is no quantum confinement effect for

10 longer $(\mathbf{I P h})_{\mathrm{n}}$ arrays, which is unexpected but can be explained in terms of alternating local

11 diatropic and paratropic ring currents. The calculations also show that the $(\mathbf{I P h})_{\mathrm{n}}$ arrays have

12 several quasi-degenerated low-lying excited states that are accessible through magnetic-

13 dipole transitions.

14 Calculations on the $\left(\mathbf{H}_{2} \mathbf{P}\right)_{\mathrm{n}}$ arrays show that local paratropic ring currents are confined in

15 the molecular moieties between the porphyrin subunits (Figure 4), whereas the porphyrin

16 rings sustain weak diatropic ring currents. The global delocalization of the $\pi$ electrons leads

17 to a small non-vanishing diatropic ring current of $6 \mathrm{nA} \mathrm{T}^{-1}$ that flows along the central $\beta-\beta$

18 C-C link, which results in a smaller HLG for the longer $\left(\mathbf{H}_{2} \mathbf{P}\right)_{\mathrm{n}}$ arrays. The small HLG leads

19 to a stronger paratropic ring currents than for the (IPh $)_{\mathrm{n}}$ arrays that do not sustain any ring-

20 current flux along the central $\beta-\beta$ C-C link, which is denoted with the dashed line in Figure

21 4. The weak global ring current of $6 \mathrm{nA} \mathrm{T}^{-1}$ for the $\left(\mathbf{H}_{2} \mathbf{P}\right)_{\mathrm{n}}$ arrays suggests that they have a 
1 global electron delocalization leading to a decreasing HLG for longer arrays in contrast to

2 the $(\mathbf{I P h})_{\mathrm{n}}$ arrays, which do not sustain any global ring current. Thus, the $(\mathbf{I P h})_{\mathrm{n}}$ arrays lack 3 global electron delocalization, which results in a size-independent HLG.

5 Figure 3. The molecular structures of the studied porphyrin $\left(\mathbf{H}_{2} \mathbf{P}\right)$ and isophlorin (IPh)

arrays. Top chart represents linear oligomers $\left(\mathbf{H}_{2} \mathbf{P}\right)_{\mathrm{n}}$ and $(\mathbf{I P h})_{\mathrm{n}}(\mathrm{n}=0-7)$, while in the bottom

part shows the square-shaped TPS and TIPhS arrays.

The $\left(\mathbf{H}_{2} \mathbf{P}\right)_{\mathrm{n}}$ arrays are diamagnetic because the strengths of the local paratropic ring currents in the naphthalene-pyrrole moieties are less than $20 \mathrm{nA} \mathrm{T}^{-1}$, which has been found to be the critical ring-current strength for closed-shell paramagnetism of porphyrinoids. The diatropic ring currents in the porphyrin rings also cancel the paramagnetic contribution to $\chi_{z z}$.

3 The $(\mathbf{I P h})_{\mathrm{n}}$ species exhibit a diamagnetic character due to same reason namely, the strength of the local paratropic ring currents in the isophlorin fragments is less than threshold value of $-20 \mathrm{nA} \mathrm{T}^{-1}$ and diatropic ring currents in the naphthalene moieties between the isophlorin rings cancel the paramagnetic contribution to $\chi_{z z}$. However, the magnetizability of $(\mathbf{I P h})_{\mathrm{n}}$
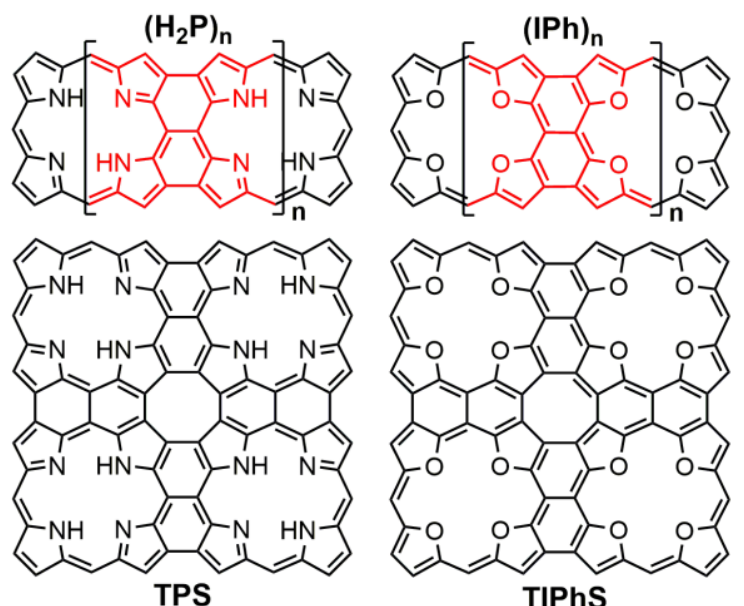

TIPhS 
1 is less diamagnetic than for the $\left(\mathbf{H}_{2} \mathbf{P}\right)_{\mathrm{n}}$ analogues $(-11$ vs. -96 a.u. for $\mathrm{n}=1,-127$ vs. -30 a.u.

2 for $\mathrm{n}=2$, etc., see Table 2). Thus, if one would be able to slightly increase the strength of the

3 local paratropic ring current in each isophlorin unit, the whole array might become para-

4 magnetic.

6 Table 2. The magnetizability ( $\chi$ in a.u.) and the HOMO-LUMO gap (HLG in eV) of the

7 studied porphyrin $\left(\mathbf{H}_{2} \mathbf{P}\right)_{\mathrm{n}}$ and isophlorin $(\mathbf{I P h})_{\mathrm{n}}$ arrays. HLG represents the smallest denom-

8 inator in the expression for $\chi_{\mathrm{p}}$. The ring-current strengths of porphyrin and isophlorin are 27

$9 \quad \mathrm{nA} \mathrm{T}^{-1}$ and $-29 \mathrm{nA} \mathrm{T}^{-1}$, respectively.

\begin{tabular}{ccccc}
\hline & \multicolumn{2}{c}{$\left(\mathbf{H}_{\mathbf{2}} \mathbf{P}\right)_{\mathbf{n}}$} & \multicolumn{2}{c}{$(\mathbf{I P h})_{\mathbf{n}}$} \\
\hline $\mathrm{n}$ & $\chi$ & $\mathrm{HLG}$ & $\chi$ & $\mathrm{HLG}$ \\
\hline 0 & -68 & 4.46 & 12 & 3.39 \\
\hline 1 & -96 & 2.82 & -11 & 2.22 \\
\hline 2 & -127 & 2.16 & -30 & 2.17 \\
\hline 3 & -158 & 1.77 & -48 & 2.14 \\
\hline 4 & -190 & 1.50 & -68 & 2.14 \\
\hline 5 & -221 & 1.28 & -87 & 2.12 \\
\hline 6 & -252 & 1.06 & -106 & 2.12 \\
\hline 7 & -283 & 0.61 & -124 & 2.11 \\
\hline
\end{tabular}

10

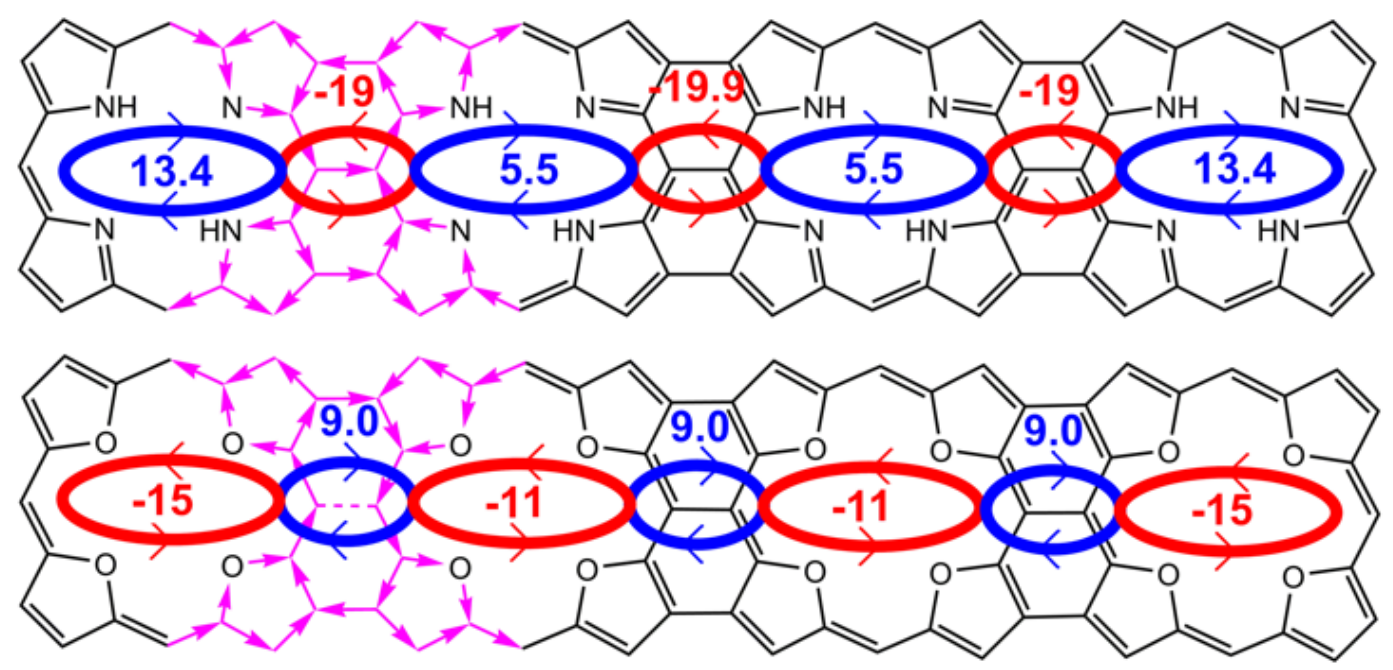


1 Figure 4. The strong local paratropic ring currents (red ellipses) and the weak diatropic ring

2 currents (blue ellipses) in the $\left(\mathbf{H}_{2} \mathbf{P}\right)_{3}$ and $(\mathbf{I P h})_{3}$ arrays (top and bottom, respectively). The

3 structure of the current-density flow is the same for the whole series of the studied mole-

4 cules $(n=1-7)$. The pink arrows show the current-density pathways, and numbers correspond

5 to strength of the ring currents (in $\mathrm{nA} \mathrm{T}^{-1}$ ).

6 We also studied the current density and magnetizability of two-dimensional (2D) quadratic

$7 \quad \mathbf{H}_{2} \mathbf{P}$ (TPS) and IPh (TIPhS) sheets (Figure 5) in which the building blocks are connected

8 by the same meso-meso, $\beta-\beta, \beta-\beta$-linking scheme as in the linear arrays. Free-base TPS

9 has been successfully synthesized and characterized by Osuka et.al., ${ }^{52,53}$ while TIPhS is

10 predicted here. TPS and TIPhS are predicted to sustain ring currents along the outer edge

11 (rim) of the molecule and in the inner eight-membered ring (hub). In contrast to the $\left(\mathbf{H}_{2} \mathbf{P}\right)_{\mathrm{n}}$

12 and $(\mathbf{I P h})_{\mathrm{n}}$ arrays, no multiple paratropic ring-current domains were found in the quadratic

13 2D sheets. The calculations show that the ring current of the hub of TPS and TIPhS is

14 paratropic (Figure 5), which agrees with previous NICS studies on TPS $^{53}$ and with studies

15 showing that hetero[8]circulenes also generally sustain a strong paratropic ring current in

16 the hub. ${ }^{54-56}$ Complexation of free-base TPS with $\mathrm{Zn}^{2+}$ yields tetra- $\mathrm{Zn}$-porhyrin

$17 \mathrm{Zn}_{4} \mathbf{T P S}^{57,58}$, where the eight inner hydrogens are replaced by four $\mathrm{Zn}^{2+}$ cations. The com-

18 plexation enhances the paratropic ring currents in the hub increasing the ring-current

19 strength from $-33 n \mathrm{nA} \mathrm{T}^{-1}$ for free-base TPS to $-65 \mathrm{nA} \mathrm{T}^{-1}$ for $\mathrm{Zn}_{4}$ TPS. The diatropic ring

20 current of $5 \mathrm{nA} \mathrm{T}^{-1}$ and $7 \mathrm{nA} \mathrm{T}^{-1}$ along the rim of TPS and $\mathrm{Zn}_{4}$ TPS, respectively, are much

21 weaker than the paratropic ones in the hub (Figure 5). However, due to the large radius of 
1 the diatropic ring current of the rim, they play a crucial role for the sign of the magnetiza-

2 bility of TPS and $\mathrm{Zn}_{4}$ TPS. The paramagnetic contribution from the strong paratropic ring

3 current of the hub with a small radius is cancelled by the diamagnetic contribution from the

4 weak diatropic ring current with a large radius. In addition, each atom brings diamagnetic

5 contributions to the magnetizability according to Pascal's rule. Therefore, free-base TPS

6 and the corresponding $\mathrm{Zn}_{4}$ TPS complex are diamagnetic with magnetizabilities of -126 a.u.

7 and -132 a.u., respectively. Due to the same reason, TIPhS sustains a paratropic ring current

8 of $-11 \mathrm{nA} \mathrm{T}^{-1}$ in the hub and a diatropic ring current of $12 \mathrm{nA} \mathrm{T}^{-1}$ along the rim leading to a

9 diamagnetic molecule with a negative magnetizability of -140 a.u.

10

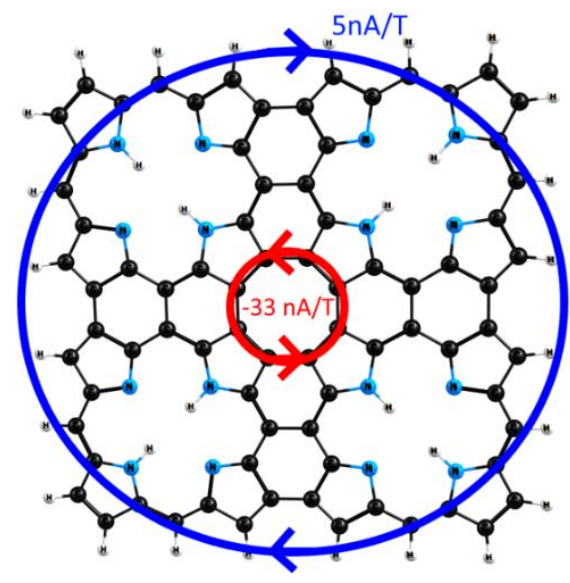

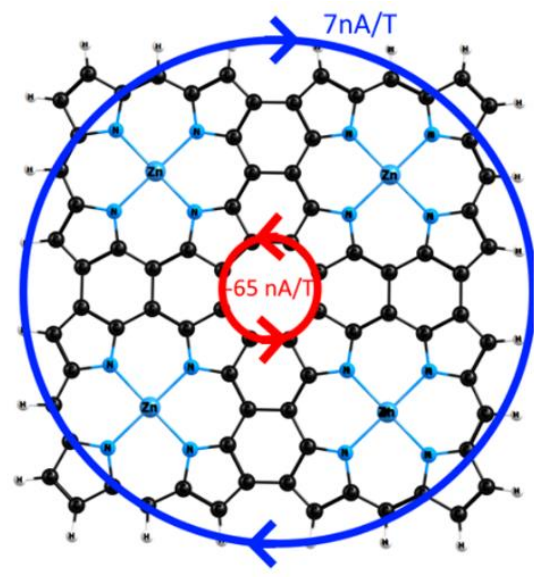

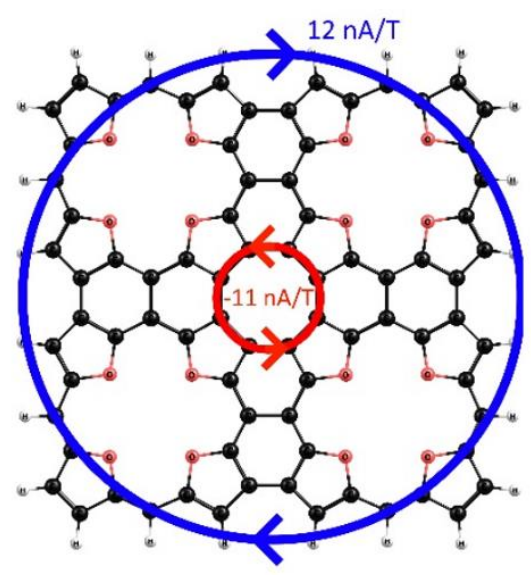

11 Figure 5. The diatropic (blue line) and paratropic (red line) magnetically induced ring cur-

12 rents in the free-base tetra-porphyrin TPS sheet (left), the $\mathrm{Zn}_{4}$ TPS sheet (middle), and in

13 the tetra-isophlorin TIPhS sheet (right).

4.4 Iso[n]phlorins. The magnetizability and ring-current strengths were calculated for isophlorin IPh (iso[4]phlorin) and a series of analogous expanded isophlorins (Figure 6). The molecular charge has been chosen such that the expanded isophlorins fulfill Hückel's rule 
1 for antiaromaticity. Isophlorin is an antiaromatic porphyrin with $28 \pi$ electrons in the por-

2 phyrin ring. Originally isophlorin was constructed from free-base porphyrin by adding two

3 inner hydrogens. ${ }^{59}$ Alternatively, one can replace the $\mathrm{NH}$ moieties with iso-electronic ox-

4 ygen atoms. ${ }^{60-62}$ The expanded isophlorins studied here consisting of 5-8 furan rings have

5 not been synthesized, whereas the synthesized cyclo-octafuran(1.1.1.1.1.1.1.1) has in-

6 verted furan rings. ${ }^{63}$ Our calculations show that the inverted (experimental) configuration

7 is $210 \mathrm{kcal} \mathrm{mol}^{-1}$ more favourable than a hypothetical circle-shaped iso[8]phlorin. For the

8 design of closed-shell paramagnetic antiaromatic molecules, we have considered a series

9 of iso[n]phlorins of the same circle-shaped topology. Calculations on this series of mole-

10 cules yield a relation between the ring-current strength, the ring-current radius and the

11 magnetizability. We have previously showed that the isophlorin ring consisting of four

12 furan rings connected via methine groups is strongly antiaromatic sustaining a paratropic

13 ring current of $-63 \mathrm{nA} \mathrm{T}^{-1}$ at the B3LYP level. ${ }^{48}$ The present M06-2X calculations yield a

14 ring-current strength of $-29 \mathrm{nA} \mathrm{T}^{-1}$ for isophlorin. The magnetizability of isophlorin calcu-

15 lated at the M06-2X level is 12 a.u. The calculated ring-current strengths and magnetiza-

16 bilities of the antiaromatic $(n=4 k)$ expanded isophlorins are summarized in Table 3 . The

17 studied isophlorins sustain strong paratropic ring currents that are of about the same

18 strength except for iso[8]phlorin, which sustains a very strong paratropic ring current of -

$19617 \mathrm{nA} \mathrm{T}^{-1}$. Since the radius increases with the number of furan rings, the magnetizability

20 also increases with n. For iso[8]phlorin the large radius of the ring current of $5.3 \AA$ and the

21 strong paratropic ring current lead to a very large positive magnetizability of 3532 a.u. 
1 Table 3. The ring-current strength $\left(\mathrm{J}\right.$ in $\left.\mathrm{nA} \mathrm{T}^{-1}\right)$, the radius of the ring current ( $\mathrm{R}$ in $\AA$ ), and

2 the average magnetizability ( $\chi$ in a.u.) of the studied iso[n]phlorins, where $\mathrm{n}$ is the number

3 of furan rings.

\begin{tabular}{lrcr}
\hline Molecule & $\mathrm{J}$ & $\mathrm{R}$ & $\chi$ \\
\hline Iso[4]phlorin & -29 & 2.0 & 12 \\
\hline Iso[5]phlorin $^{1+}$ & -73 & 2.5 & 132 \\
\hline Iso[6]phlorin $^{2+}$ & -56 & 3.7 & 140 \\
\hline Iso[7]phlorin $^{1-}$ & -45 & 4.5 & 158 \\
\hline Iso[8]phlorin & -617 & 5.3 & $3532^{\mathrm{a}}$ \\
\hline
\end{tabular}

$4{ }^{\text {a }}$ For comparison, the magnetizability of $\mathbf{c}-\mathbf{P 6} \cdot \mathbf{T} \mathbf{6}^{4+}$ is 4418 a.u. ${ }^{22}$ and the magnetizability of $\mathrm{O}_{2}\left({ }^{3} \Sigma_{g}\right)$ is 5719 a.u. at a temperature of $293 \mathrm{~K}^{64}$

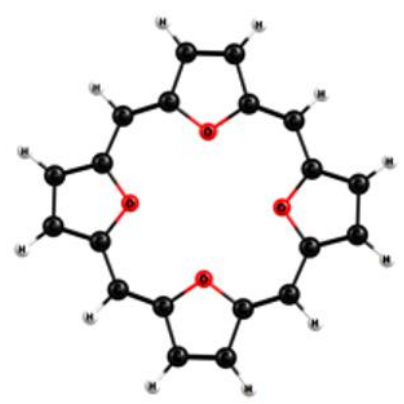

$\mathrm{IPh}(\mathrm{n}=4)$

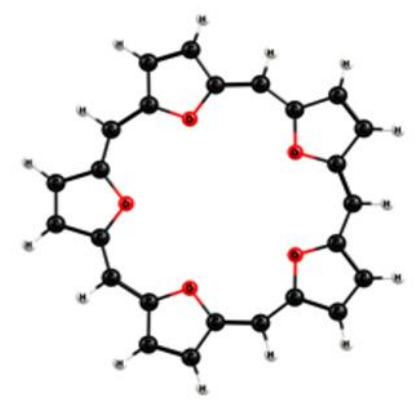

$n=5$

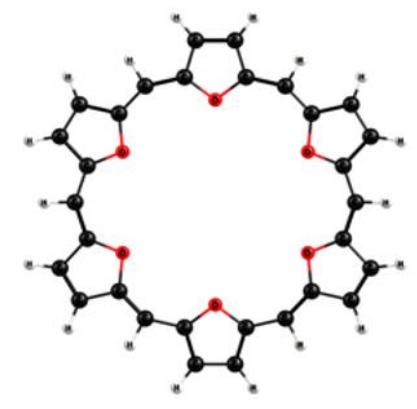

$n=6$

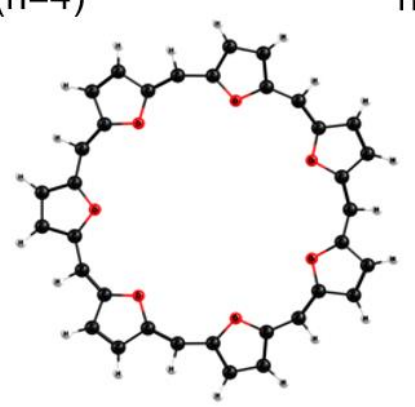

$n=7$

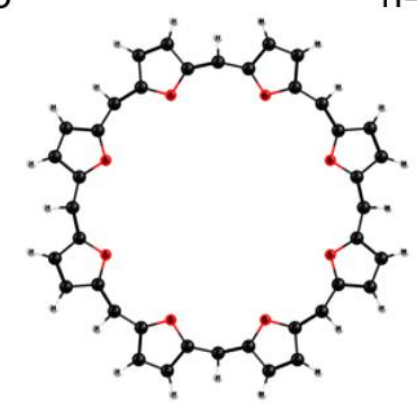

$n=8$

7 Figure 6. The molecular structures of the studied iso[n]phlorins, $n=4-8$.

4.5. Expanded porphyrinoids. We also studied orangarin, rosarin, and amethyrin, which

9 are synthesized expanded antiaromatic porphyrins whose molecular structures are shown

10 in Figure $7 .{ }^{65}$ Even though they are strongly antiaromatic, ${ }^{41}$ calculations of the magnetiza-

11 bilities show that they are diamagnetic (Table 4). The paratropic ring currents are weaker 
1 than $-20 \mathrm{nA} \mathrm{T}^{-1}$ and the radius of the ring current is not large enough to lead to closed-shell

2 paramagnetism.

3 We also studied the hypothetic circle-shaped [40]octaphyrin(1.1.1.1.1.1.1.1) molecule

4 with eight $\mathrm{NH}$ groups that consists of eight pyrrole rings connected via methine bridges.

5 The molecular structure of [40]octaphyrin(1.1.1.1.1.1.1.1) shown in Figure $7 \mathrm{~d}$ is a local

6 minimum on the potential energy surface of the singlet ground state; it lies $163 \mathrm{kcal} \mathrm{mol}^{-1}$

7 above the square-shaped [40]octaphyrin(1.1.1.1.1.1.1.1) shown in Figure 8b also contain-

8 ing eight $\mathrm{NH}$ groups, which has not been synthetized. [40]octaphyrin(1.1.1.1.1.1.1.1) with

9 inverted pyrrole rings is diamagnetic with a negative magnetzability of -67 a.u. and prac-

10 tically nonaromatic sustaining a ring current of $\mathrm{J}=3.4 \mathrm{nA} \mathrm{T}^{-1}$. In contrast, the circle-shaped

11 [40] octaphyrin(1.1.1.1.1.1.1.1) sustains a strong paratropic ring current of $-561 \mathrm{nA} \mathrm{T}^{-1}$ re-

12 sulting in a large positive magnetizability of 3244 a.u.

13 Table 4. The ring-current strength $\left(\mathrm{J}_{\text {in }} \mathrm{nA} \mathrm{T}^{-1}\right.$ ), the radius of the ring current ( $\mathrm{R}$ in $\AA$ ), and

14 the average magnetizability ( $\chi$ in a.u.) of orangarin, rosarin, amethyrin and the hypothetic

15 [40]octaphyrin(1.1.1.1.1.1.1.1).

16

\begin{tabular}{lccc}
\hline Molecule & $\mathrm{J}$ & $\mathrm{R}$ & $\chi$ \\
\hline Orangarin & -20 & 2.0 & -7.0 \\
\hline Rosarin & -18 & 2.5 & -4.9 \\
\hline Amethyrin & -14 & 2.5 & -14 \\
\hline $\begin{array}{l}\text { [40]octaphyrin(1.1.1.1.1.1.1.1) } \\
\text { (circle-shaped) }\end{array}$ & -561 & 5.3 & 3244 \\
\hline
\end{tabular}



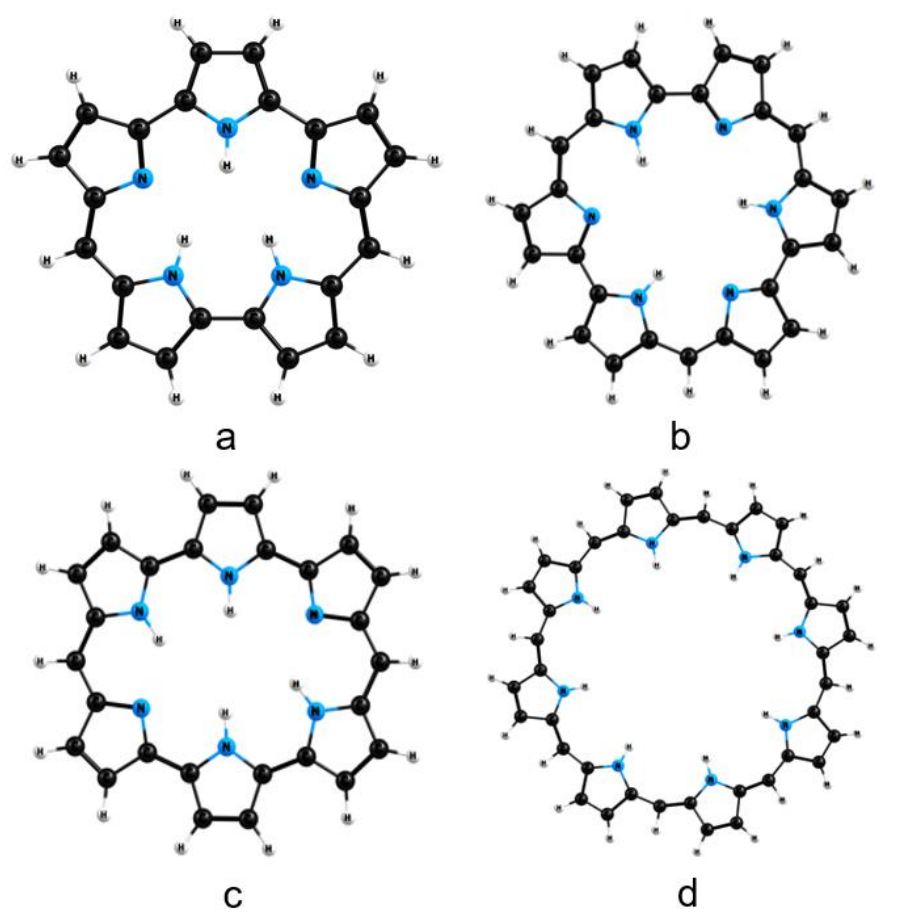

2 Figure 7. The molecular structure of the antiaromatic expanded porphyrins: a) orangarin

3 b) rosarin c) amethyrin and d) circle-shaped [40]octaphyrin(1.1.1.1.1.1.1.1).

5 The anomalously high paratropic ring currents for the hypothetical iso[8]phlorin in Figure 66 and [40]octaphyrin(1.1.1.1.1.1.1.1) in Figure 7d seem to originate from the circular to7 pology of the molecular structure. They are examples of closed-shell paramagnetic mole8 cules with a strong ring current and a large ring-current radius. Since iso[8]phlorin and 9 circle-shaped [40]octaphyrin(1.1.1.1.1.1.1.1) are strongly antiaromatic, they are high-lying 10 local minima on the potential energy surfaces of the singlet ground state. The global min-

11 imum structure has four pyrrole/furan rings with the $\mathrm{NH} / \mathrm{O}$ moiety pointing inwards and 12 four NH/O moieties pointing outwards. The synthesis of the high-lying structures is cer13 tainly challenging, whereas from the electronic structure point of view these molecules are 14 stable. 
1 4.6. Square-shaped [36] $\operatorname{coctaphyrin}(1.1 .1 .1 .1 .1 .1 .1)$ and cyclo-octafuran(1.1.1.1.1.1.1.1)

2 The previously synthesized [36]octaphyrin(1.1.1.1.1.1.1.1 ${ }^{66}$ and cyclo-octafu-

$3 \operatorname{ran}(1.1 .1 .1 .1 .1 .1 .1)^{63}$ with inverted rings shown in Figure 8 are weakly aromatic and

4 weakly antiaromatic molecules sustaining a weak diatropic $\left(2.4 \mathrm{nA} \mathrm{T}^{-1}\right)$ and weak para-

5 tropic $\left(-3.8 \mathrm{nA} \mathrm{T}^{-1}\right)$ global ring current, respectively. Cyclo-octafuran (1.1.1.1.1.1.1.1) ${ }^{63}$ is

6 a planar diamagnetic molecule with a magnetizability of -56 a.u. It sustains local diatropic

7 ring currents of 5.9 and $8.8 \mathrm{nA} \mathrm{T}^{-1}$ in the furan rings. The [36]octaphyrin(1.1.1.1.1.1.1.1 $)^{66}$

8 molecule shown in Figure 8c (it contains four NH groups in contrast to hypothetical

9 [36]octaphyrin(1.1.1.1.1.1.1.1) in Figure 8b) is also planar and diamagnetic molecule with

10 a magnetizability of -9.7 a.u. It is a weakly antiaromatic $40 \pi$ electron annulene, since the

$11 \mathrm{~N} / \mathrm{NH}$ moieties do not contribute to the global ring current due to the local ring currents in

12 the pyrrole rings. When two protons are removed from [36]octaphyrin(1.1.1.1.1.1.1.1)

13 (Figure 8d), the resulting dianion is antiaromatic with a ring-current strength of $-7 \mathrm{nAT}^{-1}$

14 and paramagnetic with a magnetizability of $\chi=9$ a.u. It is a porphyrinoid with $46 \pi$ electrons

15 showing that large molecular rings sustaining relatively weak paratropic ring currents can

16 be closed-shell paramagnetic molecules. The optimized molecular structure of the [36]oc-

17 taphyrin(1.1.1.1.1.1.1.1) dianion is ruffled, while in the single-crystal X-ray diffraction

18 measurement, the dianion with $\mathrm{C}_{6} \mathrm{~F}_{5}$ substituents in the meso-positions and with tetrabu-

19 tylammonium counterions is nearly planar. Thus, we propose that the unsubstituted [36]oc-

taphyrin(1.1.1.1.1.1.1.1) dianion is a Möbius-antiaromatic $46 \pi$ electron molecule. 

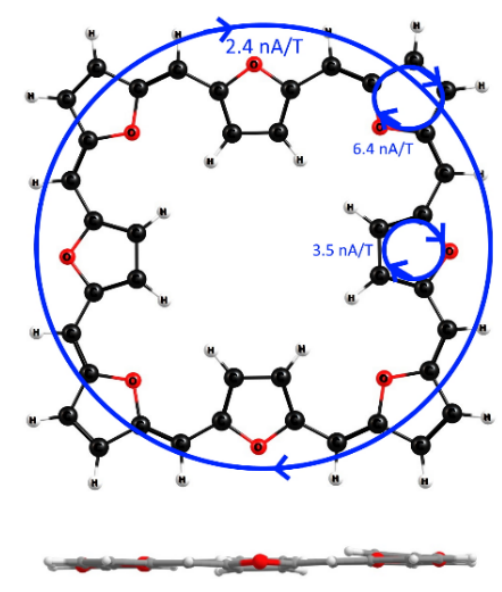

a

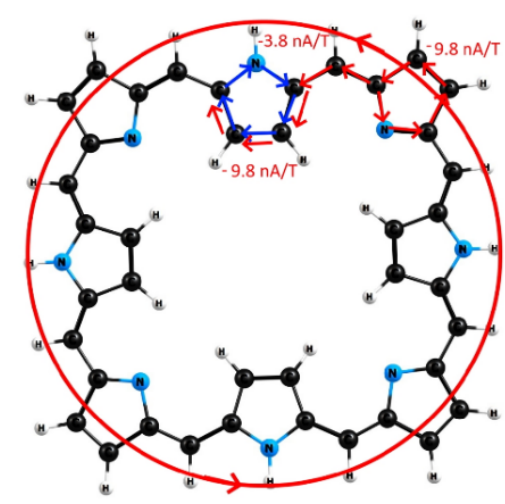

C
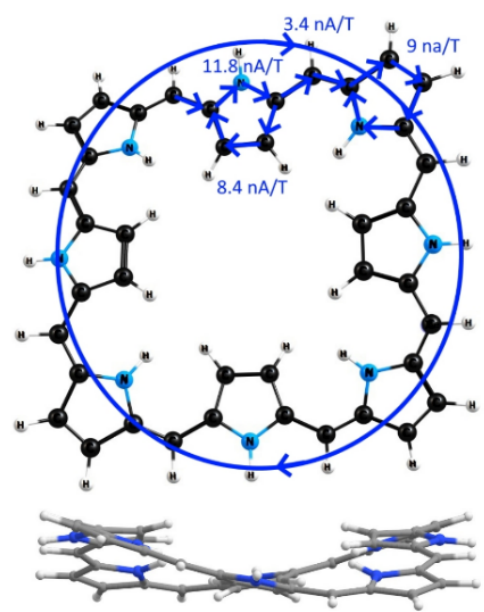

b

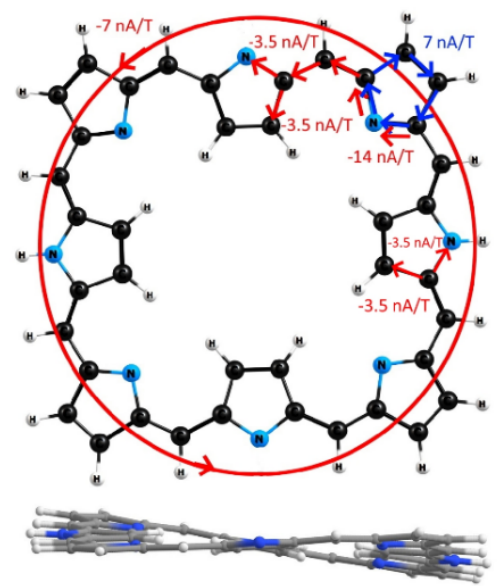

d

2 Figure 8. Current-density pathways and ring-current strengths calculated for (a) cyclo-oc-

3 tafuran(1.1.1.1.1.1.1.1), (b) hypothetical square-shaped [40] octaphyrin(1.1.1.1.1.1.1.1),

4 (c) [36]octaphyrin(1.1.1.1.1.1.1.1) and (d) [36]octaphyrin(1.1.1.1.1.1.1.1) dianion using 5 GIMIC. The side view of the molecular structures are also shown.

\section{CONCLUSIONS}

7 The present study gives a recipe for designing closed-shell paramagnetic molecular rings.

8 One of the prerequisites for closed-shell paramagnetism (CSP) of ring-shaped porphyrinoids

9 seems to be that they sustain a paratropic ring current of at least $-20 \mathrm{nA} \mathrm{T}^{-1}$. The paratropic 
1 ring current must not be cancelled by other diatropic ring currents with larger radii. Larger

2 radii of the paratropic ring current lead to stronger closed-shell paramagnetism. However,

3 the diamagnetic contribution to the magnetizability also increases with the size of the ring

4 according to Pascal's rule. For planar molecules in the $x y$ plane, the $z z$ component of the

5 magnetizability tensor can be positive due to a strong paratropic magnetically induced ring

6 current, whereas the $x x$ and $y y$ components are negative (diamagnetic) according to Pascal's

7 rule, since planar molecules do not sustain any paratropic ring currents when the magnetic

8 field is parallel to the molecular plane. Thus, closed-shell paramagnetism is a balance be-

9 tween the paramagnetic contribution to the $z z$ element of the magnetizability tensor and the

10 diamagnetic contributions from the $x x$ and $y y$ elements.

11 The reliability of the employed computational level was assessed by calculating the magnet-

12 izability of the recently synthesized tetra-cationic hexaporphyrin nanowheel $\left(\mathbf{c}-\mathbf{P 6} \cdot \mathbf{T} \mathbf{6}^{\mathbf{4}}\right)$,

13 which is a closed-shell paramagnetic molecule with an experimental magnetizability of 4418

14 a.u. ${ }^{23}$ Calculations at the M06-2X yielded a magnetizability of 3188 a.u. showing that the

15 employed computational level is able to reproduce the large positive magnetizability of $\mathbf{c}-$

$16 \mathbf{P 6} \cdot \mathbf{T 6}^{4+}$. Magnetizabilities calculated at the M06-2X level for closed-shell paramagnetic

17 molecular rings seem though to be slightly underestimated.

18 Iso[4]phlorin sustaining a ring current of $-29 \mathrm{nA} \mathrm{T}^{-1}$ with a radius of $2 \AA$ is paramagnetic

19 with a magnetizability of 12 a.u. Cyclo[8]carbon with a radius of $1.8 \AA$ sustaining a ring

20 current of $-42 \mathrm{nA} \mathrm{T}^{-1}$ is weakly paramagnetic with a magnetizability of 5 a.u. These examples

21 show that closed-shell paramagnetic molecular rings with a radius of $<2 \AA$ must sustain a

22 very strong paratropic ring current. 
1 A series of meso-meso, $\beta-\beta, \beta-\beta$-linked porphyrins (TPS) and isophlorins (IPh) extending

2 in one (arrays) and two (sheets) dimensions were studied. The linear and square-shaped

3 porphyrin and isophlorin sheets have a different topology of the ring currents. The linear

4 porphyrinoid arrays have multiple centers sustaining local diatropic and paratropic ring cur-

5 rents, whereas the squared-shaped porphyrinoid sheets sustain a paratropic ring current in

6 the hub and a diatropic ring current along the rim. Substituting the inner NH protons of TPS

7 with $\mathrm{Zn}^{2+}$ cations leads to a stronger paratropic ring current in the hub, whereas the diatropic

8 ring current along the rim is less affected.

9 The linear isophlorin arrays $(\mathbf{I P h})_{\mathrm{n}}$ sustain paratropic ring currents in each of the isophlorin

10 moieties and a diatropic ring current in the naphthalene moieties between the isophlorins.

11 The $(\mathbf{I P h})_{\mathrm{n}}$ arrays do not sustain any global ring current. The absence of global electron

12 delocalization leads to a very weak quantum confinement for the (IPh $)_{\mathrm{n}}$ arrays implying that

13 the HOMO-LUMO gap is almost independent of the number of isophlorins moieties for the

14 longer $(\mathbf{I P h})_{\mathrm{n}}$ arrays.

15 For the $\left(\mathbf{H}_{2} \mathbf{P}\right)_{\mathrm{n}}$ arrays, a global diatropic ring current through the $\beta-\beta$ links results in a smaller

16 HOMO-LUMO gap for the longer $\left(\mathbf{H}_{2} \mathbf{P}\right)_{\mathrm{n}}$ arrays. The porphyrin moieties sustain local dia-

17 tropic ring currents, whereas the linking naphthalene-pyrrole moieties sustain local para-

18 tropic ring currents. The linear arrays sustaining several local paratropic ring currents are

19 diamagnetic, because the ring-current strengths do not exceed $-20 \mathrm{nA} \mathrm{T}^{-1}$. Several paratropic

20 ring currents in the same molecule does not make them paramagnetic, because the diamag-

21 netic contribution increases also linearly with the number of atoms in the molecule. 
1 Calculations on a series of expanded isophlorins fulfilling Hückel's rule for antiaromaticity

2 show that they are paramagnetic due to the strong paratropic ring current and the large radius

3 of the ring current. The largest member of the series namely iso[8]phlorin has a magnetiza-

4 bility of 3532 a.u., because it sustains a very strong ring current of $-617 \mathrm{nA} \mathrm{T}^{-1}$ whose radius

5 is $5.3 \AA$. Calculations on expanded porphyrins fulfilling Hückel's rule for antiaromaticity

6 show that orangarin, rosarin, amethyrin are diamagnetic, since they sustain paratropic ring

7 currents that a weaker than $-20 \mathrm{nA} \mathrm{T}^{-1}$. The largest member of that series is the hypothetic

8 circle-shaped [40] octaphyrin(1.1.1.1.1.1.1.1) that sustains a strong paratropic ring current of

$9 \quad-561 \mathrm{nA} \mathrm{T}^{-1}$ whose radius is $5.3 \AA$. The circle-shaped [40]octaphyrin(1.1.1.1.1.1.1.1) mole-

10 cule is therefore strongly paramagnetic with a magnetizability of 3244 a.u.

12 AUTHOR INFORMATION

13 Corresponding Author

14 E-mail: glibar@kth.se, rashid.valiev@helsinki.fi

15 Notes

16 The authors declare no competing financial interests.

17 ACKNOWLEDGMENT

18 This work has been supported by the Olle Engkvist Byggmästare foundation (contract no.

19 189-0223) and by the Ministry of Education and Science of Ukraine (project no.

20 0117U003908). The calculations were performed with computational resources provided

21 by the High-Performance Computing Center North (HPC2N) in Umeå, Sweden, through 
1 the project "Multiphysics Modeling of Molecular Materials" SNIC 2019-2-41. The

2 GIMIC calculations were carried out using the SKIF supercomputer at the Tomsk State

3 University. This project has also been funded by the Academy of Finland through projects

41315600 and 314821. R.R.V. thanks the Tomsk Polytechnic University Competitiveness

5 Enhancement Program (VIU-RSCABS-142/2019) for support. R.T.N. thanks Russian Sci-

6 entific Foundation (18-19-00268)

ASSOCIATED CONTENT

9 Supporting Information

10 The Supporting Information is available free of charge on the ACS Publications website at

11 DOI: 10.1021/acs.jpcc.XXXXXXX. Optimized geometries (Cartesian coordinates) for all

12 studied molecules $\left(\mathrm{c}-\mathrm{P} 6 \cdot \mathrm{T}^{4+}\right.$ nanowheel, cyclo[n]carbons $(\mathrm{n}=8,12,16 \ldots 44),\left(\mathrm{H}_{2} \mathrm{P}\right)_{\mathrm{n}}(\mathrm{n}=0-$

13 7) and $(\mathrm{IPh})_{\mathrm{n}}(\mathrm{n}=0-7)$ arrays, TPS, $\mathrm{Zn}_{4}$ TPS and TIPhS nanosheets, iso[n]phlorins $(\mathrm{n}=4-8)$,

14 expanded porphyrinoids, octaphyrins and cyclo-octa furans).

15

16 REFERENCES

17 (1) Krygowski, T. M., Cyrański, M. K. Structural Aspects of Aromaticity, Chem. Rev. 2001, $18 \quad 101,1385-1420$.

19 (2) Mucsi, Z.; Viskolcz, B.; Csizmadia, I. G. A Quantitative Scale for the Degree of Aroma20 ticity and Antiaromaticity: A Comparison of Theoretical and Experimental Enthalpies of 21 Hydrogenation, J. Phys. Chem. A, 2007, 111 1123-1132. 
1 (3) Shin, J.-Y.; Kim, K. S.; Yoon, M.-C.; Lim, J. M.; Yoon, Z. S.; Osuka, A.; Kim D. Aro-

2 maticity and Photophysical Properties of Various Topology-Controlled Expanded Porphy-

3 rins, Chem. Soc. Rev. 2010, 39, 2751-2767.

4 (4) Gershoni-Poranne, R.; Stanger, A. Magnetic Criteria of Aromaticity, Chem. Soc. Rev.

$5 \mathbf{2 0 1 5}, 44,6597-6615$.

6 (5) Fujii, S.; Marqués-González, S.; Shin, J.-Y.; Shinokubo, H.; Masuda, T.; Nishino, T.;

7 Arasu, N. P.; Vázquez, H.; Kiguchi, M. Highly-Conducting Molecular Circuits Based on

8 Antiaromaticity, Nat. Commun. 2017, 8, 15984.

9 (6) Chen, Z.; Wannere, C. S.; Corminboeuf, C.; Puchta, R.; Schleyer, P. v R. Nucleus-Inde-

10 pendent Chemical Shifts (NICS) as an Aromaticity Criterion. Chem. Rev. 2005, 105, 3842-

113888.

12 (7) Sundholm, D.; Fliegl, H.; Berger, R. J. F. Calculations of Magnetically Induced Current

13 Densities: Theory and Applications, WIREs Comput. Mol. Sci. 2016, 6, 639-678.

14 (8) Yamashina, M.; Tanaka, Y.; Lavendomme, R.; Ronson, T. K.; Pittelkow M.; Nitschke J.

15 R. An Antiaromatic-Walled Nanospace, Nature 2019, 574, 511-515.

16 (9) Pedersen, S. K.; Eriksen, K.; Karaush-Karmazin, N. N.; Minaev, B.; Ågren, H.; Barysh-

17 nikov, G. V.; Pittelkow, M. Anti-Aromatic vs. Induced Paratropicity: Synthesis and Interro-

18 gation of a Dihydro-Diazatrioxa[9]circulene With a Proton Placed Directly Above the Cen-

19 tral Ring. Angew. Chem. Int. Ed. 2020, 59, 5144-5150.

20 (10) Baryshnikov, G. V.; Valiev, R. R.; Kuklin, A. V.; Sundholm, D.; Ågren, H. Cy21 clo[18]carbon: Insight into Electronic Structure, Aromaticity, and Surface Coupling. J. Phys.

22 Chem. Lett. 2019, 10, 6701-6705. 
1 (11) Berger, R. J. F.; Viel, A. The symmetry principle of antiaromaticity. Zeitschrift für

2 Naturforschung B. 2020, 75, 327-339.

3 (12) Feixas, F.; Matito, E.; Poater, J.; Solà, M. Quantifying Aromaticity with Electron Delo-

4 calisation Measures. Chem. Soc. Rev. 2015, 44, 6434-6451.

5 (13) Van Vleck J. H. The theory of electric and magnetic susceptibilities, Oxford, Clarendon

6 Press, 1932.

7 (14) Lazzeretti P. General Connections Among Nuclear Electromagnetic Shieldings and Po-

8 larizabilities. Adv. Chem. Phys. 1989, 75, 507-549.

9 (15) Lazzeretti, P.; Malagoli, M.; Zanasi R. Computational Approach to Molecular Magnetic

10 Properties by Continuous Transformation of the Origin of the Current Density. Chem. Phys.

11 Lett. 1994, 220, 299-304.

12 (16) Lazzeretti P. Ring Currents. Prog. Nucl. Mag. Res. Sp. 2000, 36, 1-88.

13 (17) Guo, R.; Uddin, M. N.; Price, L. S.; Price S. L. The Calculation of the Diamagnetic

14 Susceptibility Tensors of Organic Crystals: from Coronene to Pharmaceutical Polymorphs.

15 J. Phys. Chem. A 2020, 124, 1409-1420.

16 (18) Hegstrom, R. A.; Lipscomb, W. N. Paramagnetism in Closed-Shell Molecules. Rev.

17 Mod. Phys. 1968, 40, 354-358.

18 (19) Fowler, P. W.; Steiner, E. Paramagnetic Closed-Shell Molecules: the Isoelectronic series

$19 \mathrm{CH}^{+}$, BH and $\mathrm{BeH}^{-}$. Mol. Phys. 1991, 74, 1147-1158.

20 (20) Sauer, S. P. A.; Enevoldsen, T.; Oddershede, J. Paramagnetism of Closed Shell Dia-

21 tomic Hydrides with Six Valence Electrons. J. Chem. Phys. 1993, 98, 9748-9757. 
1 (21) Ruud, K.; Helgaker, T.; Bak, K. L.; Jørgensen, P.; Olsen, J. Accurate Magnetizabilities

2 of the Isoelectronic Series $\mathrm{BeH}^{-}, \mathrm{BH}$, and $\mathrm{CH}^{+}$. The MCSCF-GIAO Approach. Chem. Phys.

$31995,195,157-169$.

4 (22) Carrington, A. The Temperature-Independent Paramagnetism of Permanganate and Re5 lated Complexes, Mol. Phys. 1960, 3, 271-275.

6 (23) Peeks, M. D.; Claridge, T. D. W.; Anderson, H. L. Aromatic and Antiaromatic Ring

7 Currents in a Molecular Nanoring. Nature, 2017, 541, 200-203.

(24) Valiev, R. R.; Fliegl, H.; Sundholm, D. Closed-shell paramagnetic porphyrinoids.

9 Chem. Commun. 2017, 53, 9866-9869.

10 (25) Valiev, R. R.; Fliegl, H.; Sundholm D. Optical and magnetic properties of antiaromatic 11 porphyrinoids. Phys. Chem. Chem. Phys. 2017, 19, 25979-25988.

12 (26) Valiev, R. R.; Baryshnikov, G. V.; Sundholm, D.; Relations Between the Aromaticity 13 and Magnetic Dipole Transitions in the Electronic Spectra of Hetero[8]circulenes, Phys.

14 Chem. Chem. Phys. 2018, 20, 30239-30246.

15 (27) Helgaker, T.; Coriani, S.; Jørgensen, P.; Kristensen, K.; Olsen, J.; Ruud, K. Recent 16 Advances in Wave Function-Based Methods of Molecular-Property Calculations, Chem. 17 Rev. 2012, 112, 543-631.

18 (28) Ruud, K.; Helgaker, T.; Bak, K. L.; Jørgensen, P.; Jensen, H. J. Aa. Hartree-Fock limit

19 Magnetizabilities from London Orbitals, J. Chem. Phys. 1993, 99, 3847-3859.

20 (29) Ruud, K.; Skaane, H.; Helgaker, T.; Bak, K. L.; Jørgensen, P. Magnetizability of Hy21 drocarbons. J. Am. Chem. Soc. 1994, 116, 10135-10140. 
1 (30) Dahle, P.; Ruud, K.; Helgaker, T.; Taylor P. R. Molecular Polarizabilities and Magnet-

2 izabilities. Theor. Comp. Chem. 1999, 6, 147-188.

3 (31) Stevens, R. M.; Pitzer, R. M.; Lipscomb, W. N. Perturbed Hartree-Fock Calculations.

4 I. Magnetic Susceptibility and Shielding in the LiH Molecule. J. Chem. Phys. 1963, 38, 5505560.

6 (32) Pascal, P. Recherches Magnetochimiques. Ann. Chim. Phys. 1910, 19, 5-70.

7 (33) Zhao, Y.; Truhlar, D. G. The M06 Suite of Density Functionals for Main Group Ther-

8 mochemistry, Thermochemical Kinetics, Noncovalent Interactions, Excited States, and

9 Transition Elements: Two New Functionals and Systematic Testing of Four M06-class Func-

10 tionals and 12 other Functionals. Theor. Chem. Acc. 2008, 120, 215-241

11 (34) Weigend, F.; Ahlrichs, R. Balanced Basis Sets of Split Valence, Triple Zeta Valence and 12 Quadruple Zeta Valence Quality for H to Rn: Design and Assessment of Accuracy. Phys.

13 Chem. Chem. Phys. 2005, 7, 3297-3305

14 (35) Baryshnikov, G. V.; Valiev, R. R.; Lie, Q.; Lie, C.; Xie, Y.; Ågren, H. Computational

15 Study of Aromaticity, ${ }^{1} \mathrm{H}$ NMR Spectra and Intermolecular Interactions of Twisted Thia16 norhexaphyrin and Its Multiply Annulated Polypyrrolic Derivatives. Phys. Chem. Chem.

17 Phys. 2019, 21, 25334-25343.

18 (36) Valiev, R. R.; Fliegl, H.; Sundholm, D. Bicycloaromaticity and Baird-type bicycloaro-

19 maticity of dithienothiophene-bridged [34] octaphyrins. Phys. Chem. Chem. Phys. 2018, 20, $20 \quad 17705-17713$. 
1 (37) Wolinski, K.; Hilton J. F.; Pulay, P. Efficient Implementation of the Gauge-Independent

2 Atomic Orbital Method for NMR Chemical Shift Calculations. J. Am. Chem. Soc. 1990, 112, $3 \quad 8251-8260$.

4 (38) Fisch, M. J.; Trucks, G. W.; Schlegel, H. B.; Scuseria, G. E.; Robb, M. A.; Cheeseman,

5 J. R.; Scalmani, G.; Barone, V.; Petersson, G. A.; Nakatsuji, H.; et al. Gaussian 16, Revision 6 A.03, Gaussian, Inc., Wallingford CT, 2016.

7 (39) Jusélius, J.; Sundholm, D. Calculation of Current Densities Using Gauge-Including

8 Atomic Orbitals. J. Chem. Phys. 2004. 121, 3952-3963.

9 (40) Gauge-Including Magnetically Induced Currents (GIMIC) program is distributed on 10 GitHub platform and can be downloaded through the link: https://github.com/qmcur11 rents/gimic.

12 (41) Granovsky, A. A. Firefly version 8.0.0, http://classic.chem.msu.su/gran/firefly/in13 dex.html.

14 (42) Valiev, R. R.; Benkyi, I.; Konyshev, Y. V.; Fliegl, H.; Sundholm, D. Computational 15 Studies of Aromatic and Photophysical Properties of Expanded Porphyrins. J. Phys. Chem. 16 A 2018, 122, 4756-4767.

17 (43) Herges, R. Topology in Chemistry: Designing Möbius Molecules. Chem. Rev. 2006, $18 \quad 106,4820-4842$.

19 (44) Kaiser, K.; Scriven, L. M.; Schulz, F.; Gawel, P.; Gross, L.; Anderson, H. L. An Sp20 Hybridized Molecular Carbon Allotrope, Cyclo[18]Carbon. Science. 2019, 365 (6459), $21 \quad 1299-1301$. 
1 (45) Fowler, P. W.; Mizoguchi, N.; Bean, D. E.; Havenith, R. W. A. Double Aromaticity and

2 Ring Currents in All-Carbon Rings. Chem. - A Eur. J. 2009, 15, 6964-6972.

3 (46) Baryshnikov, G. V.; Valiev, R. R.; Kuklin, A. V.; Sundholm, D.; Ågren H. Cyclo[18]car-

4 bon: Insight into Electronic Structure, Aromaticity, and Surface Coupling. J. Phys. Chem.

5 Lett. 2019, $10(21), 6701-6705$.

6 (47) Fliegl, H.; Sundholm, D. Aromatic Pathways of Porphins, Chlorins, and Bacteriochlo-

7 rins. J. Org. Chem. 2012, 77, 3408-3414.

8 (48) Valiev, R. R.; Fliegl, H.; Sundholm, D. Insights into Magnetically Induced Current

9 Pathways and Optical Properties of Isophlorins. J. Phys. Chem. A, 2013, 117, 9062-9068.

10 (49) Fliegl, H.; Tauber, S.; Lehtonen, O.; Sundholm D. The Gauge Including Magnetically

11 Induced Current Method. Phys. Chem. Chem. Phys. 2011, 13, 20500-20518.

12 (50) Tsuda, A.; Osuka, A. Fully Conjugated Porphyrin Tapes with Electronic Absorption

13 Bands That Reach into Infrared. Science 2001, 293, 79-82.

14 (51) Ikeda, T.; Aratani, N.; Osuka, A. Synthesis of Extremely $\pi$-Extended Porphyrin Tapes

15 from Hybrid meso-meso Linked Porphyrin Arrays: An Approach Towards the Conjugation

16 Length. Chemistry-An Asian Journal. 2009, 4, 1248-1256.

17 (52) Nakamura, Y.; Aratani, N.; Furukawa, K.; Osuka, A. Synthesis and Characterizations

18 of Free Base and $\mathrm{Cu}(\mathrm{II})$ Complex of a Porphyrin Sheet. Tetrahedron 2008, 64, 11433-11439.

19 (53) Tanaka, T.; Osuka A. Conjugated Porphyrin Arrays: Synthesis, Properties and Applica-

20 tions for Functional Materials, Chem. Soc. Rev. 2015, 44, 943-969. 
1 (54) Baryshnikov, G. V.; Valiev, R. R.; Karaush, N. N.; Minaev, B. F. Aromaticity of the

2 Planar Hetero[8]circulenes and Their Doubly Charged Ions: NICS and GIMIC Characteri-

3 zation. Phys. Chem. Chem. Phys. 2014, 16, 15367-15374.

4 (55) Baryshnikov, G. V.; Valiev, R. R.; Karaush, N. N.; Sundholm, D.; Minaev, B. F. Aro5 maticity of the Doubly Charged [8]Circulenes. Phys. Chem. Chem. Phys. 2016, 18, 898068992.

7 (56) Baryshnikov, G. V.; Valiev, R. R.; Cherepanov, V. N.; Karaush-Karmazin, N. N.;

8 Minaeva, V. A.; Minaev, B. F.; Ågren, H. Aromaticity and Photophysics of Tetrasila- and 9 Tetragerma-Annelated Tetrathienylenes as New Representatives of the Hetero[8]circulene 10 Family. Phys. Chem. Chem. Phys. 2019, 21, 9246-9254.

11 (57) Nakamura, Y.; Aratani, N.; Shinokubo, H.; Takagi, A.; Kawai, T.; Matsumoto, T.; Yoon, 12 Z. S.; Kim, D. Y.; Ahn, T. K.; Kim, D.; Muranaka, A.; Kobayashi, N.; Osuka A. A Directly 13 Fused Tetrameric Porphyrin Sheet and Its Anomalous Electronic Properties That Arise from 14 the Planar Cyclooctatetraene Core. J. Am. Chem. Soc. 2006, 128, 4119-4127.

15 (58) Nakamura, Y.; Aratani, N.; Osuka, A. Experimental and Theoretical Investigations into 16 the Paratropic Ring Current of a Porphyrin Sheet. Chem. Asian J. 2007, 2, 860-866.

17 (59) Woodward, R. B. Totalsynthese des Chlorophylls. Angew. Chem., 1960, 72, 651-662.

18 (60) Reddy, J. S.; Anand, V. G. Planar Meso Pentafluorophenyl Core Modified Isophlorins.

19 J. Am. Chem. Soc., 2008, 130, 3718-3719.

20 (61) Reddy, B. K.; Basavarajappa, A.; Ambhore, M. D.; Anand V. G. Isophlorinoids: The 21 Antiaromatic Congeners of Porphyrinoids. Chem. Rev., 2017, 117, 3420-3443; 
1 (62) Chatterjee, T.; Srinivasan, A.; Ravikanth, M.; Chandrashekar, T. K. Smaragdyrins and

2 Sapphyrins Analogues. Chem. Rev., 2017, 117, 3254-3328.

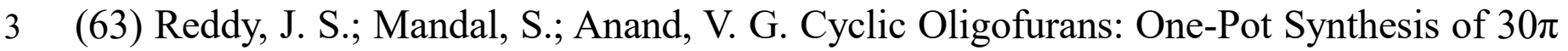
4 and $40 \pi$ Expanded Porphyrinoids. Organic letters, 2006, 8, 5541-5543.

5 (64) Glick, R. E. On The Diamagnetic Susceptibility of Gases J. Phys. Chem. 1961, 65, $6 \quad 1552-1555$.

7 (65) Jasat, A.; Dolphin, D. Expanded Porphyrins and Their Heterologs. Chem. Rev. 1997, $897,2267-2340$.

9 (66) Cha, W. Y.; Soya, T.; Tanaka, T.; Mori, H.; Hong, Y.; Lee, S.; Park, K. H.; Osuka, A.;

10 Kim, D. Multifaceted [36]octaphyrin (1.1.1.1.1.1.1.1): deprotonation-induced switching

11 among nonaromatic, Möbius aromatic, and Hückel antiaromatic species. Chem. Commun.

12 2016, 52, 6076-6078. 